\title{
As estratégias para avaliação de aprendizagem empregadas em estudantes de enfermagem de ensino superior: revisão integrativa
}

The strategies for learning assessment applied to nursing students of higher education: integrative review

Las estrategies de evaluación del aprendizaje aplicadas en estudiantes de enfermería de educación superior: revisión integradora

Beatriz Queiroz Ribeiro ORCID: https://orcid.org/0000-0002-0603-0804 Universidade Estadual de Londrina, Brasi E-mail:biaqribeiro@gmail.com

Carla Fernanda Tiroli ORCID: https://orcid.org/0000-0002-0974-9689 Universidade Estadual de Londrina, Brasil E-mail: carla_tiroli@yahoo.com.br

Natacha Bolorino

ORCID: https://orcid.org/0000-0002-3039-2987 Universidade Estadual de Londrina, Brasil E-mail: natachabolorino@hotmail.com

Ana Beatriz Floriano de Souza ORCID: https://orcid.org/0000-0002-4721-5370 Universidade Estadual de Londrina, Brasil E-mail: anabia.enfe@gmail.com

Alberto Durán González

ORCID: https://orcid.org/0000-0002-4203-9400 Universidade Estadual de Londrina, Brasil E-mail: betoduran80@gmail.com

Mara Solange Gomes Dellaroza ORCID: https://orcid.org/0000-0002-7869-540X Universidade Estadual de Londrina, Brasil E-mail: dellaroza@uel.br

Franciely Midori Bueno de Freitas ORCID: https://orcid.org/0000-0003-0815-2765 Universidade Estadual de Londrina, Brasil E-mail: franmidori@hotmail.com

Natalia Marciano de Araújo Ferreira ORCID: https://orcid.org/0000-0002-5802-6188 Universidade Estadual de Londrina, Brasil E-mail: natty_fdj@hotmail.com

Laís Cristina Gonçalves Ribeiro ORCID: https://orcid.org/0000-0002-4522-3297

Universidade Estadual de Londrina, Brasil E-mail: lcg.enf@hotmail.com

Rafaela Marioto Montanha ORCID: https://orcid.org/0000-0002-7237-0110

Universidade Estadual de Londrina, Brasil E-mail: rafaela.montanha@hotmail.com

Denise Andrade Pereira ORCID: https://orcid.org/0000-0003-1141-8229 Universidade Estadual de Londrina, Brasil

E-mail: demeier01@gmail.com

Ariane Sabina Stieven

ORCID: 0000-0002-8082-9067

Universidade Estadual de Londrina, Brasil

E-mail: ariane_stieven@gmail.com

Flávia Meneguetti Pieri

ORCID: https://orcid.org/0000-0003-1239-2550 Universidade Estadual de Londrina, Brasil E-mail: fpieri@uel.br 


\title{
Resumo
}

Foi realizada uma revisão integrativa, com objetivo de identificar quais as estratégias para a avaliação de aprendizagem estão sendo empregadas aos estudantes de enfermagem de ensino superior. A pesquisa foi norteada pela estratégia PICO: qual a efetividade de intervenções avaliativas disponíveis na literatura científica sobre as estratégias para avaliação de aprendizagem aplicadas aos estudantes de enfermagem do nível superior? A busca por estudos foi realizada entre janeiro e fevereiro de 2019 nas seguintes bases de dados: LILACS, Medline, Scielo, BDENF, PubMed, CINAHL e Scopus. Foram incluídas 24 referências e identificadas 8 estratégias de avaliação. A maioria citou avaliação em práticas, seguida de feedback, avaliação teórica, avaliação interpares, autoavaliação, avaliação de trabalho em grupo e por último avaliação qualitativa. Observou-se que $75 \%(n=18)$ dos estudos inclusos foram realizados fora do cenário brasileiro. Dos estudos internacionais, 20,8\% estão concentrados no Reino Unido, $12,5 \%$ em Hong Kong e 12,5\% na Irlanda. No Brasil, os estudos se concentraram na região sudeste, com 12,5\% em São Paulo e 8,3\% em Minas Gerais. Quanto ao método utilizado, 66\% dos artigos foram de abordagem qualitativa, $20,8 \%$ utilizaram de método quantitativo e 20,8\% empregaram método misto, com abordagem quantitativa e qualitativa. Por fim, este estudo identificou como os principais instrumentos de avaliação usados no ensino superior de enfermagem: avaliação em práticas, feedback, portfólio, avaliação teórica, avaliação interpares, autoavaliação, avaliação de trabalho em grupo e avaliação qualitativa. Sugere-se a realização de estudos nacionais, a fim de ampliar as discussões e aprimorar as técnicas de avaliações aplicadas.

Palavras-chave: Aprendizagem; Estudantes de enfermagem; Métodos de avaliação; Educação superior; Ensino.

\begin{abstract}
An integrative review was carried out, aiming to identify which strategies to learning assessment are being applied to nursing students of higher education. The research was guided by the PICO strategy: what is the effectiveness of evaluative interventions available in the scientific literature on the strategies for learning assessment applied to nursing students at university level? The search for studies was performed between January and February 2019 in the following databases: LILACS, Medline, Scielo, BDENF, PubMed, CINAHL and Scopus. 24 references were included, and eight assessment strategies were identified. Most cited assessment in practices, followed by feedback, theoretical assessment, peer evaluation, self-assessment, group work assessment and, finally, qualitative assessment. It was noted that $75 \%(n=18)$ of the included studies were carried out outside the Brazilian scenario. Of the international studies, $20.8 \%$ are concentrated in the United Kingdom, 12.5\% in Hong Kong and 12.5\% in Ireland. In Brazil, studies were concentrated in the southeast region, with $12.5 \%$ in São Paulo and $8.3 \%$ in Minas Gerais. As for the method used, $66 \%$ of the papers had a qualitative approach, $20.8 \%$ used a quantitative method and $20.8 \%$ used a mixed method with a quantitative and qualitative approach. Finally, this study identified as the main assessment tools used in higher education in nursing: assessment in practices, feedback, portfolio, theoretical assessment, peer evaluation, self-assessment, group work assessment and qualitative assessment. It is suggested to accomplish national studies, in order to broaden the discussions and improve the applied assessment techniques.
\end{abstract}

Keywords: Learning; Nursing students; Assessment methods; Higher education; Teaching.

\section{Resumen}

Se realizó una revisión integradora aspirando identificar qué estrategias de evaluación de aprendizajes están siendo aplicadas a los estudiantes de enfermería en la educación superior. La investigación se guió por la estrategia PICO: ¿cuál es la efectividad de las intervenciones evaluativas disponibles en la literatura científica sobre las estrategias de evaluación del aprendizaje aplicadas a estudiantes de enfermería a nivel universitario? La búsqueda de estudios se realizó entre enero y febrero de 2019 en las siguientes bases de datos: LILACS, Medline, Scielo, BDENF, PubMed, CINAHL y Scopus. Se incluyeron 24 referencias y se identificaron 8 estrategias de evaluación. La mayoría citó la evaluación en prácticas, seguida de feedback, evaluación teórica, evaluación por pares, autoevaluación, evaluación del trabajo en grupo y, finalmente, evaluación cualitativa. Se observó que el $75 \%(n=18)$ de los estudios incluidos se realizaron fuera del escenario brasileño. De los estudios internacionales, el 20,8\% se concentra en Reino Unido, el $12,5 \%$ en Hong Kong y el 12,5\% en Irlanda. En Brasil, los estudios se concentraron en la región sureste, con 12,5\% en São Paulo y 8,3\% en Minas Gerais. En relación con el método utilizado, el 66\% de los artículos fueron de enfoque cualitativo, el 20,8\% utilizó un método cuantitativo y el 20,8\% utilizó un método mixto, con enfoque cuantitativo y cualitativo. Finalmente, este estudio identificó como las principales herramientas de evaluación utilizadas en la educación superior en enfermería: evaluación en prácticas, feedback, portafolio, evaluación teórica, evaluación por pares, autoevaluación, evaluación del trabajo en grupo y evaluación cualitativa. Se sugiere realizar estudios nacionales con el fin de ampliar las discusiones y mejorar las técnicas de evaluación aplicadas.

Palabras clave: Aprendizaje; Estudiantes de enfermería; Métodos de evaluación; Educación superior; Ensenãnza. 


\section{Introdução}

Para as instituições de ensino superior, obter resultados satisfatórios na formação dos discentes tem sido um desafio a ser enfrentado. Nos cursos voltados para a área da saúde, tem-se o enfoque em uma educação crítico-reflexiva que busque repostas aos desafios expostos pela população. Sendo assim, as diretrizes nacionais de ensino apontam para a importância da formação voltada para o mercado de trabalho, requerendo profissionais comprometidos com a responsabilidade social e a cidadania, atendendo a promoção da saúde integral das pessoas que requerem cuidados (Pascon, Otrenti \& Mira, 2018).

No que concerne ao curso de enfermagem, o processo de avaliação do discente é extremamente complexo e demonstra-se como um dos maiores desafios de implementação e manutenção curricular, exigindo dos alunos e professores capacitação constante (Pascon, Otrenti \& Mira, 2018).

Segundo as Diretrizes Curriculares Nacionais (DCN), o processo avaliativo dos estudantes de enfermagem deve contemplar o domínio cognitivo, avaliação de competências e habilidades (Costa et al., 2018).

No modelo tradicional de ensino, o processo avaliativo é visto por meio da concepção de medida, com uso de nota para mensurar o conteúdo apreendido e sendo operacionalizado em forma de provas e exames, o que unicamente não contribui para a aprendizagem articulada às necessidades de formação profissional do estudante (Cavalcante \& Mello, 2015).

A forma de avaliar os graduandos de enfermagem tem acompanhado a trajetória da educação em geral, movendo-se de uma proposta tradicional para formas mais participativas e democráticas em que o aluno é o protagonista do processo ensino-aprendizagem (Bernardi et al., 2014; Fontes et al., 2021).

Podemos citar algumas formas de avaliação de ensino identificadas na literatura como: portfólio, avaliação em práticas clínicas, avaliação teórica, feedback, avaliação interpares, avaliação em sala de aula, apresentações pessoais e em grupo, planos de desenvolvimento pessoal, tutoriais, discussões online, avaliação de competências, avaliação de trabalho em grupo, autoavaliação e prova teórica de progressão.

A literatura apresenta estudos direcionados sobre a evolução da educação e preocupações referentes às avaliações no processo educacional.

Porém, poucos estudos refletem sobre as práticas avaliativas no curso de graduação em enfermagem, objeto desse estudo. Desta forma, objetivo de identificar quais as estratégias para avaliação de aprendizagem estão sendo empregadas aos estudantes de enfermagem de ensino superior.

\section{Método}

Trata-se de uma revisão integrativa da literatura de abordagem qualitativa, sua finalidade é de realizar a síntese e análise dos dados presentes na literatura para desenvolver uma explicação mais abrangente de um fenômeno específico, com propósitos teóricos ou de intervenção (Santos et al., 2013; Pereira, Shitsuka, Parreira \& Shitsuka, 2018). As etapas que conduziram esta pesquisa foram: elaboração da pergunta norteadora; coleta de dados; avaliação dos dados; análise e interpretação dos dados; apresentação dos resultados e conclusões (Mendes, Silveira \& Galvão, 2008).

A pergunta norteadora foi formulada, utilizando a estratégia PICO, que é um acrônimo usado para representar os quatro termos que a compõem: o Paciente ou Problema (P), a Intervenção (I), a Comparação (C) e o Desfecho (O - do inglês: Outcomes) (Santos, Pimenta \& Nobre, 2007).

Partindo-se destes pressupostos, formou-se a seguinte pergunta: qual a efetividade de intervenções avaliativas disponíveis na literatura científica sobre as estratégias para avaliação de aprendizagem aplicadas aos estudantes de enfermagem 
do nível superior? Sendo que, o $\mathbf{P}$ estudantes de enfermagem do nível superior; I intervenções educacionais individual ou coletiva; $\mathbf{C}$ não foi abordado, pois não era objetivo deste estudo comparar intervenções; $\mathbf{O}$ aprendizagem.

Os artigos foram selecionados entre o período de 1976 a 2018, os critérios de inclusão foram artigos disponíveis na íntegra em qualquer período ou ano, em português, inglês e/ou espanhol, que abordavam as formas de avaliação dos estudantes de enfermagem de ensino superior. Foram excluídos Teses, dissertações, trabalhos de conclusão de curso, capítulos de livros, relatos de casos, artigos provenientes de revisões ou reflexões teóricas e que abordassem a aplicação de escalas aos alunos.

A busca por estudos foi realizada entre janeiro e fevereiro de 2019 nas seguintes bases de dados: Literatura LatinoAmericana e do Caribe em Ciências da Saúde (LILACS), Medical Literature Analysis and Retrieval System Online (Medline), Scientific Electronic Library Online (Scielo), Banco de Dados em Enfermagem (BDENF), National Library of Medicine National Institutes of Health (PubMed), Cumulative Index to Nursing and Allied Health Literature (CINAHL) e Scopus Preview (Scopus).

Os descritores controlados são provenientes dos Descritores em Ciência da Saúde (DeCS) e Medical Subject Headings Terms (MeSH). Sendo assim, para a busca nas bases LILACS, Medline, Scielo e BDENF, foram utilizados os descritores: Graduandos de enfermagem; estudantes de enfermagem; avaliação educacional e avaliação de desempenho. Para CINAHL, PubMed e Scopus foram: Students, nursing e education measurement. Utilizou-se ainda as palavras-chave: Avaliação bidimensional; avaliação de competência; bacharelado de enfermagem; aluno de enfermagem; competency assessment $e$ student performance appraisal.

$\mathrm{Na}$ base de dados LILACS, Medline, Scielo e BDENF, os termos foram combinados da seguinte forma: ((Graduandos de enfermagem OR alunos de enfermagem OR estudantes de enfermagem OR bacharelado de enfermagem) AND (avaliação bidimensional OR avaliação educacional OR avaliação de desempenho OR avaliação de competencia)).

Os termos combinados nas bases de dados PubMed, CINAHL e Scopus foram: ((Students, nursing OR Nursing Students OR Pupil Nurse) AND (Education Measurement OR Competency Assessment OR Student Performance Appraisal)).

Todos os artigos buscados foram hospedados no software StArt - State of the Art through Systematic Review, ferramenta computacional de apoio a revisões sistemáticas que oferece suporte às etapas de planejamento, execução, análise final de dados e visualização dos resultados (Fabbri et al., 2016).

Para avaliação dos dados, recorreu-se ao PRISMA (Preferred Reporting Items for Systematic and Meta-Analyses) para a triagem dos estudos, contendo as seguintes etapas: 1) identificação; 2) triagem; 3) elegibilidade e 4) inclusão (Moher et al., 2009).

$\mathrm{Na}$ etapa de identificação, a amostra de estudos foi extraída, armazenada e quantificada, eliminando-se os estudos duplicados com o auxílio do StArt. Na triagem, seis revisores realizaram a seleção dos artigos por meio da leitura do título e resumo. Na etapa de elegibilidade, procedeu-se a leitura na integra dos estudos pré-selecionados. A leitura foi feita em pares por quatro revisores de forma independente, e em caso de discordância acionou-se o quinto revisor. Os revisores não foram cegos para a autoria dos manuscritos. Por último, a inclusão, dessa etapa resultou a amostra final dos estudos elegíveis.

Após a avaliação inicial dos artigos, eles foram comparados entre si, buscando-se critérios de semelhança ou de diferença. A análise e interpretação dos dados foram realizadas de forma organizada e sintetizada por meio da elaboração de um quadro sinóptico que compreendeu os seguintes itens: identificação do estudo, referência/Classificação-Qualis, local do estudo, objetivo e formas de avaliação. 


\section{Resultados}

Na busca inicial, encontrou-se um total de 14743 publicações. Ao exportar essas publicações para o programa StArt, foram identificados e excluídos 9582 artigos duplicados, restando o montante de 5161 documentos.

A seleção por título e resumo resultou em 109 referências, após avaliação na íntegra foram excluídos 85 artigos, por se tratarem de publicações que versavam acerca de escalas de avaliação aplicadas aos estudantes ou que tratavam de estudantes de enfermagem e outros cursos. Assim, no total, foram incluídas 24 referências nesta revisão (Figura 1).

Figura 1. Fluxograma dos artigos considerados na revisão bibliográfica com abordagem integrativa. Brasil, 2019.

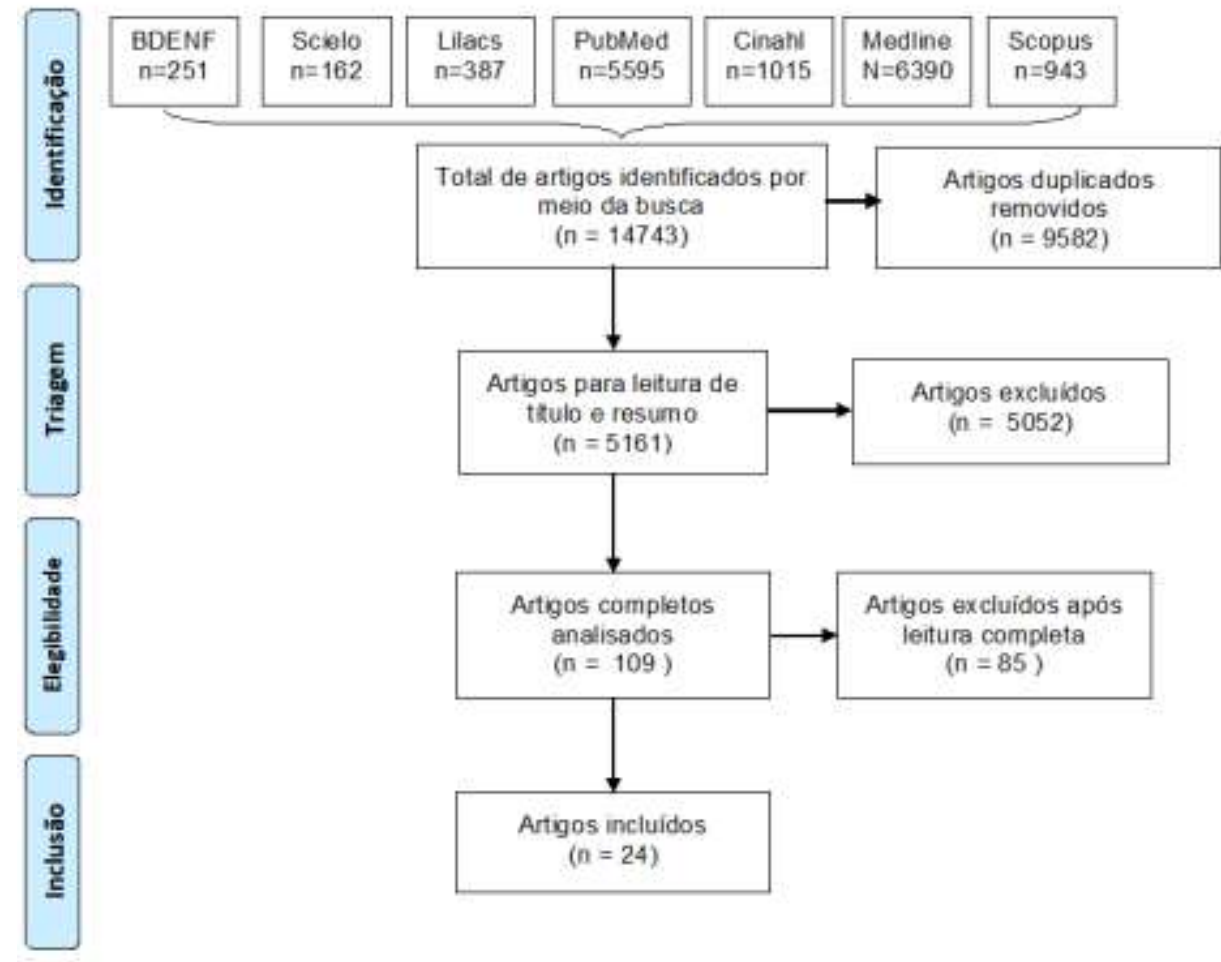

Fonte: Adaptado do diagrama PRISMA-P 2009 Statemen, (Moher et al., 2009).

Os artigos selecionados na revisão estão entre o período de 1976 a 2018, sendo que 66\% (n=14) foram publicados a partir de 2009. Observou-se que 75\% $(n=18)$ dos estudos inclusos foram realizados fora do cenário brasileiro. Dos estudos internacionais, 20,8\% (n=5; E5, E6, E8, E9, E20) estão concentrados no Reino Unido, 12,5\% (n=3; E2, E3, E15) em Hong Kong e 12,5\% (n=3; E14, E17, E21) na Irlanda. No Brasil, os estudos se concentraram na região sudeste com 12,5\% (n=3; E1, E12, E24) em São Paulo e 8,3\% (n=2; E4, E10) em Minas Gerais.

Quanto ao método utilizado, 66\% ( $\mathrm{n}=14)$ dos artigos foram de abordagem qualitativa, 20,8\% ( $\mathrm{n}=5)$ utilizou de método quantitativo e $20,8 \%$ (n=5) empregou método misto com abordagem quantitativa e qualitativa. O Quadro 1 demonstra as estratégias utilizadas para avaliação de aprendizagem dos estudantes de enfermagem de ensino superior. 
Research, Society and Development, v. 10, n. 3, e18610313238, 2021

(CC BY 4.0) | ISSN 2525-3409 | DOI: http://dx.doi.org/10.33448/rsd-v10i3.13238

Quadro 1. Síntese geral dos estudos elegidos na revisão integrativa, relacionados às formas de avaliação aplicadas aos estudantes de enfermagem de ensino superior. Brasil, 2019.

\begin{tabular}{|c|c|c|c|c|}
\hline ID & Referência/Classificação Qualis & Local do estudo & Objetivo & Formas de avaliação \\
\hline E1 & $\begin{array}{l}\text { KURCGANT, P. } \\
1976 . \\
\text { A2 }\end{array}$ & $\begin{array}{l}\text { Brasil } \\
\text { São Paulo }\end{array}$ & $\begin{array}{l}\text { Obter um perfil da atuação dos estudantes na prática de campo comparando-o } \\
\text { com o padrão de desempenho determinado pela disciplina. }\end{array}$ & $\begin{array}{l}\text { Boletim de avaliação: } \\
\text { Autoavaliação. } \\
\text { Compilado de observações (meio } \\
\text { complementar de avaliação). }\end{array}$ \\
\hline E2 & $\begin{array}{l}\text { TIWARI, T.C.; TANG, C., } 2003 . \\
\text { A2 }\end{array}$ & $\begin{array}{lll}\text { Hong } & \text { Kong } & - \\
\text { China } & & \end{array}$ & $\begin{array}{l}\text { The purpose of this study was to evaluate the effectiveness of } \\
\text { portfolio assessment in enhancing student learning. }\end{array}$ & Portfólio \\
\hline E3 & $\begin{array}{l}\text { TIWARI, A. et al., } 2005 . \\
\text { A2 }\end{array}$ & $\begin{array}{ll}\text { Hong } & \text { Kong }- \\
\text { China } & \end{array}$ & $\begin{array}{l}\text { The purpose of this study was to explore the perceptions about what students } \\
\text { learned and how they learned during their clinical practicum, and to examine the } \\
\text { role played by assessment in influencing student learning. }\end{array}$ & Avaliação em práticas clínicas \\
\hline E4 & $\begin{array}{l}\text { REIS, D.C.; MATOS, S.S.; PRADO, } \\
\text { M.L., 2005. } \\
\text { B1 }\end{array}$ & $\begin{array}{l}\text { Brasil } \\
\text { Minas Gerais }\end{array}$ & $\begin{array}{l}\text { Identificar e analisar as representações sociais sobre avaliação feita pelos } \\
\text { docentes no processo de ensino-aprendizagem dos discentes. }\end{array}$ & Avaliação teórica \\
\hline E5 & $\begin{array}{l}\text { MCMULLAN, M., } 2006 . \\
\text { A1 }\end{array}$ & Reino Unido & $\begin{array}{l}\text { To determine nursing students' perceptions on the use and effectiveness of } \\
\text { portfolios in their education as well as their perceptions on the use of the } \\
\text { portfolio as both an assessment and learning tool in clinical placement. }\end{array}$ & $\begin{array}{l}\text { Portfólio } \\
\text { Avaliação somativa e formativa }\end{array}$ \\
\hline E6 & $\begin{array}{l}\text { DEARNLEY, C.A., } 2007 . \\
\text { A2 }\end{array}$ & Reino Unido & $\begin{array}{l}\text { This paper reports on a study designed to evaluate the implementation of self- } \\
\text { assessment among student health care practitioners }\end{array}$ & $\begin{array}{l}\text { Autoavaliação } \\
\text { Feedback }\end{array}$ \\
\hline E7 & $\begin{array}{l}\text { SILVA, C.M.S.L.M.D.; TANJI S., } 2008 . \\
\text { B2 }\end{array}$ & $\begin{array}{l}\text { Brasil } \\
\text { Rio de Janeiro }\end{array}$ & $\begin{array}{l}\text { Conhecer a percepção dos estudantes de enfermagem diante de um novo olhar } \\
\text { sobre a prática avaliativa mediadora de aprendizagem. }\end{array}$ & $\begin{array}{l}\text { Avaliação somativa } \\
\text { Avaliação qualitativa }\end{array}$ \\
\hline
\end{tabular}


Research, Society and Development, v. 10, n. 3, e18610313238, 2021

(CC BY 4.0) | ISSN 2525-3409 | DOI: http://dx.doi.org/10.33448/rsd-v10i3.13238

\begin{tabular}{|c|c|c|c|c|}
\hline E8 & $\begin{array}{l}\text { DUERS, L.E.; BROWN, N., } \\
2009 . \\
\text { B1 }\end{array}$ & Reino Unido & $\begin{array}{l}\text { The study aim was to explore student nurses experiences of formative } \\
\text { assessment. }\end{array}$ & $\begin{array}{l}\text { Avaliação formativa: } \\
\text { Feedback } \\
\text { Avaliação interpares }\end{array}$ \\
\hline E9 & $\begin{array}{l}\text { KOH, L.C.; LUKE, A. } 2009 . \\
\text { A1 }\end{array}$ & Reino Unido & $\begin{array}{l}\text { The overall aim of this research was to explore how academic staff experience, } \\
\text { understand and interpret the process of formative assessment and feedback of } \\
\text { theoretical assessment. }\end{array}$ & $\begin{array}{l}\text { Avaliação formativa: Apresentações } \\
\text { individuais e de grupo, planos de } \\
\text { desenvolvimento pessoal, tutoriais, } \\
\text { avaliação interpares e discussão online } \\
\text { Feedback }\end{array}$ \\
\hline E10 & $\begin{array}{l}\text { FRIEDRICH, D.B.C. et al., } 2010 . \\
\text { A1 }\end{array}$ & $\begin{array}{l}\text { Brasil } \\
\text { Juiz de fora - } \\
\text { Minas Gerais }\end{array}$ & Analisar a utilização do portfólio pela comunidade acadêmica. & $\begin{array}{l}\text { Avaliação teórica } \\
\text { Portfólio }\end{array}$ \\
\hline E11 & $\begin{array}{l}\text { VAISMORADI, M.; PARSA-YEKTA, } \\
\text { Z., } 2011 . \\
\text { B2 }\end{array}$ & Irã & $\begin{array}{l}\text { Explore the comprehension and experiences of Iranian nursing students } \\
\text { regarding evaluation process in both classroom and clinical setting. }\end{array}$ & $\begin{array}{l}\text { Avaliação em práticas clínicas } \\
\text { Avaliação em sala de aula } \\
\text { Avaliação teórica }\end{array}$ \\
\hline E12 & $\begin{array}{l}\text { BRACCIALLI, L.; OLIVEIRA, M., } \\
2011 . \\
\text { A2 }\end{array}$ & $\begin{array}{l}\text { Brasil } \\
\text { Marília } \quad \text {-São } \\
\text { Paulo }\end{array}$ & $\begin{array}{l}\text { Identificar concepções de avaliação dos docentes no } \\
\text { Exercício de Avaliação da Prática Profissional. }\end{array}$ & Avaliação em práticas clínicas \\
\hline E13 & $\begin{array}{l}\text { RYAN, M., } 2011 . \\
\text { B2 }\end{array}$ & $\begin{array}{l}\text { Nova York - } \\
\text { Estados Unidos }\end{array}$ & $\begin{array}{l}\text { Determine the effectiveness of using the portfolio through the opinion of a } \\
\text { faculty and student. }\end{array}$ & Portfólio \\
\hline E14 & $\begin{array}{l}\text { BRADSHAW, C. et al., } 2012 . \\
\text { B3 }\end{array}$ & Irlanda & $\begin{array}{l}\text { Describe the experiences of student nurses using the } \\
\text { competency assessment framework in practice. }\end{array}$ & $\begin{array}{l}\text { Avaliação em práticas clínicas } \\
\text { Avaliação de competências }\end{array}$ \\
\hline
\end{tabular}


Research, Society and Development, v. 10, n. 3, e18610313238, 2021

(CC BY 4.0) | ISSN 2525-3409 | DOI: http://dx.doi.org/10.33448/rsd-v10i3.13238

\begin{tabular}{|c|c|c|c|c|}
\hline E15 & $\begin{array}{l}\text { SHIU, A.T.Y. et al., } 2012 . \\
\text { B1 }\end{array}$ & $\begin{array}{l}\text { Hong Kong- } \\
\text { China }\end{array}$ & $\begin{array}{l}\text { To examine baccalaureate nursing students' perceptions of peer assessment in } \\
\text { assessing individual contributions to a group project. }\end{array}$ & Avaliação interpares \\
\hline E16 & $\begin{array}{l}\text { PLAKHT, Y., et al., } 2013 . \\
\text { B1 }\end{array}$ & Israel & $\begin{array}{l}\text { To evaluate the level of feedback provided to nursing students during clinical } \\
\text { practice and investigate their association with related outcomes, such us clinical } \\
\text { performance, self-evaluation of achievements and contribution of the practice to } \\
\text { the professional skills. }\end{array}$ & $\begin{array}{l}\text { Feedback } \\
\text { Autoavaliação }\end{array}$ \\
\hline E17 & $\begin{array}{l}\text { CASSIDY, I. et al., } 2012 . \\
\text { B2 }\end{array}$ & Irlanda & $\begin{array}{l}\text { The purpose of this study was to evaluate clinical competence assessment in BSc } \\
\text { nursing registration education programmes. }\end{array}$ & $\begin{array}{l}\text { Avaliação em práticas clínicas } \\
\text { Avaliação de competências }\end{array}$ \\
\hline E18 & $\begin{array}{l}\text { SMITH, M.; ROGERS, J., } 2014 . \\
\text { A1 }\end{array}$ & Austrália & $\begin{array}{l}\text { This article reports on research that compared undergraduate nursing students } \\
\text { perceptions of two different approaches to the grading of group work assessment. }\end{array}$ & Avaliação de trabalho em grupo. \\
\hline E19 & $\begin{array}{l}\text { SPENCE, W., et al., } 2004 . \\
\text { B1 }\end{array}$ & Inglaterra & $\begin{array}{l}\text { Determine the practice teachers early experience of the portfolio approach to } \\
\text { practice assessment including its use in: student self-evaluation, guiding the } \\
\text { compilation of evidence and the development of the student's professional } \\
\text { practice. }\end{array}$ & Portfólio \\
\hline E20 & $\begin{array}{l}\text { MCMAHON-PARKES, } \\
\text { CHAPMAN, L.; JAMES, J., } 2015 . \\
\text { A1 }\end{array}$ & Reino Unido & $\begin{array}{l}\text { The aim of this study was to evaluate patients', carers', mentors' and adult field } \\
\text { nursing students' experiences of patients and carers. }\end{array}$ & Feedback \\
\hline E21 & $\begin{array}{l}\text { BURKE, E. et al., } 2016 . \\
\text { A1 }\end{array}$ & Irlanda & $\begin{array}{l}\text { To explore Irish preceptors' experience of using a competence tool to assess } \\
\text { undergraduate nursing students' clinical competence. }\end{array}$ & $\begin{array}{l}\text { Avaliação em práticas clínicas } \\
\text { Avaliação de competências }\end{array}$ \\
\hline E22 & $\begin{array}{l}\text { VAE, K.J.U. et al., } 2018 . \\
\text { A1 }\end{array}$ & Noruega & $\begin{array}{l}\text { The aim of this study was to investigate student-preceptor dyads and their } \\
\text { experiences of mid-course discussions } \\
\text { and final assessment. }\end{array}$ & $\begin{array}{l}\text { Avaliação em práticas clínicas } \\
\text { Autoavaliação } \\
\text { Feedback }\end{array}$ \\
\hline
\end{tabular}


Research, Society and Development, v. 10, n. 3, e18610313238, 2021

(CC BY 4.0) | ISSN 2525-3409 | DOI: http://dx.doi.org/10.33448/rsd-v10i3.13238

\begin{tabular}{|c|c|c|c|c|}
\hline E23 & $\begin{array}{l}\text { MARTÍNEZ, J.C., } 2017 . \\
\text { B2 }\end{array}$ & Colômbia & $\begin{array}{l}\text { El objetivo fue caracterizar las prácticas evaluativas docentes del programa de } \\
\text { enfermería. }\end{array}$ & $\begin{array}{l}\text { Autoavaliação } \\
\text { Co-avaliação } \\
\text { Heteroavaliação } \\
\text { Avaliação formativa } \\
\text { Avaliação somativa } \\
\text { Avaliação diagnóstica } \\
\text { Avaliação teórica }\end{array}$ \\
\hline E24 & $\begin{array}{l}\text { PASCON, D.M; OTRENTI, E.; MIRA, } \\
\text { V.L., } 2018 . \\
\text { A2 }\end{array}$ & $\begin{array}{l}\text { Brasil } \\
\text { São Paulo }\end{array}$ & $\begin{array}{l}\text { Conhecer o desempenho e a percepção dos estudantes de enfermagem na prova } \\
\text { de progressão e verificar a existência de relação entre o desempenho e a série em } \\
\text { curso e entre o desempenho e o grau de dificuldade da prova. }\end{array}$ & Prova teórica de Progressão \\
\hline
\end{tabular}

Fonte: Dados da pesquisa. 
A fim de melhor ilustrar as principais estratégias de avaliação utilizadas em prol do ensino superior no curso de enfermagem, apresentamos o Quadro 2.

Quadro 2. Estratégias de avaliação associadas ao ensino da enfermagem. Brasil, 2019.

\begin{tabular}{|l|l|}
\hline Instrumento de avaliação & Estudos incluídos na revisão \\
\hline Avaliação em práticas & E3; E8; E11; E12; E14; E17; E21; E22. \\
\hline Feedback & E6; E8; E9; E16; E20; E22. \\
\hline Portfólio & E2; E5; E10; E13; E19. \\
\hline Avaliação teórica & E4; E10; E11; E23; E24. \\
\hline Avaliação interpares & E8; E9; E15. \\
\hline Autoavaliação & E1; E22; E23. \\
\hline Avaliação de trabalho em grupo & E18. \\
\hline Avaliação qualitativa & E7. \\
\hline
\end{tabular}

Fonte: Dados da pesquisa.

Ao analisar os artigos encontrados nesta revisão, percebeu-se evidências de que há potencialidades, fragilidades e desafios a serem atribuídos aos instrumentos de avaliação, como a seguir.

Foi demonstrado que a avaliação em práticas pode avaliar conhecimentos, habilidades e atitudes, importantes para a avaliação da totalidade discente, ao mesmo tempo os estudantes e docentes relatam que a avaliação pode ser injusta, por haver fatores que podem afetar a decisão do professor no momento da avaliação. O desafio seria identificar as necessidades individuais de aprendizagem aos alunos para que o ensino seja focado nas dificuldades dos estudantes.

Por meio do feedback é possibilitado fomentar o progresso discente, devido a criar informações sobre as dificuldades, potencialidades e pontos de melhoria dos estudantes. O feedback no formato escrito muito utilizado no cenário internacional dificulta o entendimento dos estudantes sobre o resultado do feedback. Como desafio tem-se a promoção de um feedback assertivo para que contribua à evolução do estudante.

O portfólio promove o crescimento pessoal e profissional do estudante por favorecer a autorreflexão crítica sobre o seu aprendizado teórico-prático, no entanto, os estudos demonstraram que os estudantes não recebem uma orientação coerente para a construção do portfólio, gerando muitas dúvidas, por isso, um desafio para os docentes que utilizam desse instrumento é orientar a construção de maneira assertiva aos discentes.

A avaliação teórica é uma avaliação individual do aluno com potencial para que o docente conheça e possa intervir nas dificuldades do aluno. As fragilidades estão na utilização da nota como punição e em não estimular a capacidade de raciocínio a depender da forma de elaboração do instrumento. O desafio, portanto, estaria na elaboração da prova, para que a mesma reflita questões aplicáveis a prática do enfermeiro.

Conjuntamente à avaliação interpares é possível que os colegas façam críticas construtivas e assim, promover a confiança do aluno, no entanto, este instrumento enfrenta algumas dificuldades, pois as críticas são mais aceitas quando vindas de preceptores e não de colegas. O desafio seria a instrução mais assertiva aos alunos sobre o instrumento e a sua utilização desde início do curso para que os estudantes internalizem e percebam o significado de seu uso. 
A autoavaliação possibilita a autocrítica do estudante, enquanto também os estudantes não se sentem preparados em realizá-la e tanto docentes como estudantes enfrentam dificuldades no momento de preencher a ferramenta de autoavaliação, os professores focam na qualidade das tarefas enquanto estudantes focam na quantidade. O desafio para este instrumento é utilizar ferramentas padronizadas para a autoavaliação com orientação sobre a utilização.

Por meio da avaliação de trabalho em grupo o estudante exercita a habilidade de trabalho em equipe, no entanto, ao avaliar o coletivo pode penalizar os bons alunos. Este instrumento não dispunha de desafio.

Do mesmo modo, a avaliação qualitativa só dispunha de potencialidades, como interação teórico-prático, o aluno é responsável pela construção e evolução do seu próprio aprendizado, estimula o pensamento crítico e desvaloriza a soma de notas.

\section{Discussão}

O avaliar um discente vai além de quantificá-lo e sim promover o entendimento do conteúdo e ajudá-lo a ter uma absorção do conhecimento significativo e qualitativamente, por meio do julgamento do desempenho do mesmo. Pode-se afirmar, então, que a avaliação não deve ser considerada um fim e sim um processo (Carvalho \& Rosa, 2013).

Portanto a avaliação está interligada ao processo de ensino-aprendizagem, que nada mais é que um método que envolve vários sujeitos e permite o desenvolvimento e construção de novos conhecimentos ao estudante (Bauermann, Garcia \& Pastorio, 2020).

Contudo, o quadro atual do ensino superior no Brasil segue a metodologia tradicional e tecnicista, na qual a avaliação assume a incumbência classificatória, disciplinadora e autoritária. Contrapondo a metodologia libertadora, em que a avaliação tem função diagnóstica, a qual busca crescimento e avanço do discente, usado no processo de ensino-aprendizagem (Wiebusch \& Lima, 2018).

Historicamente o ensino superior da área da saúde seguia o modelo biomédico, no qual o ensino era voltado à doença. Atualmente vive-se uma transição educacional, em que os cursos superiores da saúde seguem as necessidades da população. E para que esse modelo se solidifique, as Diretrizes Curriculares Nacionais para a Graduação de Enfermagem (DCN/ENF), promulgadas em 2001, que norteiam as Projetos Pedagógicos de Curso (PPC) os quais fomentam a educação para formação de um profissional crítico, reflexivo, generalista, humanista e criativo (Garanhani, 2016; Parecer no 3, 2001).

Para formar enfermeiros com aptidão técnica e política, providos de raciocínio, percepção e sensibilidade para as demandas da vida, do mundo, do trabalho e da sociedade, qualificando-os para intervir em situações de incertezas e instabilidades, observou-se a necessidade de uma avaliação por competências, uma vez que o graduando de enfermagem deve ser avaliado como um todo (Garanhani, 2016).

Para avaliar as competências se faz uso da avaliação formativa, o qual avalia o processo de aprendizagem do graduando, em que se leva em consideração a comparação do aluno com ele mesmo promovendo a autoavaliação, é passível de negociações para melhora do desenvolvimento promovendo a aprendizagem.

A avaliação formativa utiliza de instrumentos como a avaliação em práticas, cujo objetivo é avaliar a capacidade do aluno em vincular a teoria à prática promovendo o pensamento crítico, baseada em habilidades cognitivas como o saber-ser e o saber-fazer no campo da prática (Díaz, Pretty \& González, 2013).

Já o feedback, no qual os discentes recebem comentários avaliativos dos docentes, colegas do grupo de práticas ou até mesmo dos funcionários do setor. Contudo existe o feedback oportuno, que o mais adequado logo após a avaliação teórica, 
Research, Society and Development, v. 10, n. 3, e18610313238, 2021

(CC BY 4.0) | ISSN 2525-3409 | DOI: http://dx.doi.org/10.33448/rsd-v10i3.13238

uma vez que permite uma troca de ideias entre o professor e o aluno (Guerrero-Aragón, Chaparro-Serrano \& Garcia-Perdomo, 2017).

Acrescentado aos instrumentos de avaliação se tem o portfólio reflexivo, o qual permite a reformulação do conhecimento, de forma autônoma, liberal e de acordo com a consciência do indivíduo. Promovendo ao estudante o protagonismo do próprio aprendizado. Ao docente pertence a incumbência de estimular a curiosidade e facilitar o processo de ensino aprendizagem e avaliação (Cesário, Ribeiro, Dias, Rothebarth \& Lima, 2016).

E por fim, ainda falando de instrumentos de avaliação formativa, as avaliações teóricas ainda permeiam as metodologias ativas. Com objetivo de credibilizar um conhecimento teórico adquirido em aula, com questões discursivas ou objetivas. E os paradigmas para os alunos desse instrumento são o fracasso e lidar com recuperação. Contudo há dificuldade na elaboração e resolução das provas.

\section{Conclusão}

Ao analisar os artigos encontrados nesta revisão, identificou-se os principais instrumentos de avaliação usado no ensino superior de enfermagem elencados em avaliação em práticas, feedback, portfólio, avaliação teórica, avaliação interpares, autoavaliação, avaliação de trabalho em grupo e avaliação qualitativa. Percebeu-se evidências de que há potencialidades, fragilidades e desafios a serem atribuídos aos instrumentos de avaliação.

Evidenciou-se uma carência em publicações nacionais sobre estratégias de avaliação utilizadas em estudantes de enfermagem de ensino superior. Nesse sentido, sugere-se a realização de estudos adicionais a fim de ampliar as discussões e aprimorar as técnicas de avaliações aplicadas.

\section{Referências}

Bauermann, K. F., Garcia, C. A. X., \& Pastorio, E. (2020). Inovação no ensino superior: um estudo comparativo sobre a educação inovadora em um curso de graduação. Revista Monografias Ambientais, 19(6). https://.org/10.5902/2236130843400.

Bernardi, M. C. et al. (2014). Avaliação da aprendizagem na formação do enfermeiro: Uma reflexão sobre sua trajetória no Brasil. História de enfermagem: Revista eletrônica, 5(2), 298-309. http://www.here.abennacional.org.br/here/vol5num2artigo10.pdf.

Braccialli, L. A. D., \& Oliveira, M. A. C. D. (2011). Concepções de avaliação de desempenho em um currículo orientado por competência. Revista da Escola de Enfermagem da USP, 45(5), 1221-1228.

Bradshaw, C. et al. (2012). Nursing students' views of clinical competence assessment. British Journal of Nursing, 21(15), 923-927.

Burke, E., Kelly, M., Byrne, E., Chiardha, T. U., Mc Nicholas, M., \& Montgomery, A. (2016). Preceptors' experiences of using a competence assessment tool to assess undergraduate nursing students. Nurse Education in Practice, 17, 8-14.

Carvalho, A. C. R., \& Rosa, B. L. (2013, setembro). Papel do professor frente a aprendizagem: processo avaliativo no ensino-aprendizagem. Anais da Jornada de Didática e do Seminário de Pesquisa do CEMAD, Londrina, PR, Brasil, 2 e 1.

Cassidy, I. et al. (2012). Preceptors' views of assessing nursing students using a competency based approach. Nurse Education in Practice, 12(6), 346-351.

Cavalcante, L. P. F., \& Mello, M. A. (2015). Avaliação da aprendizagem no ensino de graduação em saúde: concepções, intencionalidades, reflexões. Avaliação, 20(2), 423-442. https://www.scielo.br/scielo.php?pid=S141440772015000200423\&script=sci_abstract\&tlng=pt.

Cesário, J. B., Ribeiro, M. R. R., Dias, R. B. F., Rothebarth, A. P., \& Lima, L. P. S. (2016). Portfólio reflexivo como estratégia de avaliação formativa. Revista Baiana de Enfermagem, 30(1), 356-364.

Costa, D. A. S. et al. (2018). Diretrizes curriculares nacionais das profissões da Saúde 2001-2004: análise à luz das teorias de desenvolvimento curricular. Interface, 22(67), 1183-1195. http://www.scielo.br/scielo.php?script=sci_arttext\&pid=S141432832018000401183\&lng=en\&nrm=iso.

Dearnley, C. A., \& Meddings, F. S. (2007). Student self-assessment and its impact on learning-A pilot study. Nurse Education Today, 27(4), 333-340.

Díaz, A. H., Pretty, M. I., \& González, M. C. (2013). Opinião dos estudantes de graduação em enfermagem da Faculdade de Ciências da Saúde da Universidade Autônoma de Chile, Temuco, na prática clínica. Ciencia y Enfermería, 19(1), 131-144. https://scielo.conicyt.cl/pdf/cienf/v19n1/art_12.pdf. 
Research, Society and Development, v. 10, n. 3, e18610313238, 2021

(CC BY 4.0) | ISSN 2525-3409 | DOI: http://dx.doi.org/10.33448/rsd-v10i3.13238

Duers, L. E., \& Brown, N. (2009). An exploration of student nurses' experiences of formative assessment. Nurse Education Today, 29(6), 654-659.

Fabbri, S. et al. (2016). Improvements in the StArt tool to better support the systematic review process. Anais da Conferência Internacional sobre Avaliação e Avaliação em Engenharia de Software, Limerick, Munster, Irlanda, 20.

Friedrich, D. B. de C., Gonçalves, A. M. C., Sá, T. S. Sanglard, L. R., Duque, D. R., \& de Oliveira, G. M. A. (2010). O portfólio como avaliação: análise de sua utilização na graduação de enfermagem. Revista Latino-Americana de Enfermagem, 18(6), 1123-1130.

Fontes, F. L. L. et al. (2021). Utilização de metodologias ativas no curso de graduação em Enfermagem: uma oportunidade de superação do modelo de ensino tradicional. Research, Society and Development, 10 (1). https://.org/10.33448/rsd-v10i1.11774

Garanhani, M. L. (2016). Os desafios na formação do Enfermeiro no século XXI. RECOM, 6(3). http://dx..org/10.19175/recom.v6i3.1638.

Guerrero-Aragón, S. C., Chaparro-Serrano, M. F., \& Garcia-Perdomo, A. A. (2017). Evaluación por competencias en salud: revisión de literatura. Educación y Educadores, 20(2), 211-225. 10.5294/edu.2017.20.2.3.

Koh, K., \& Luke, A. (2009). Authentic and conventional assessment in Singapore schools: An empirical study of teacher assignments and student work. Assessment in Education: Principles, Policy \& Practice, 16(3), 291-318.

Kurcgant, P. (1976). Sistema de avaliação de alunos em prática de campo em administração aplicada à enfermagem. Revista da Escola de Enfermagem da USP, 10(1), 101-137.

Martínez Royert, J. C. (2017). Practicas evaluativas del proceso de atención de enfermería: una visión de docentes y estudiantes. Revista Cuidarte, 8(1), 14591475 .

McMahon-Parkes, K., Chapman, L., \& James, J. (2016). The views of patients, mentors and adult field nursing students on patients' participation in student nurse assessment in practice. Nurse Education in Practice, 16(1), 202-208.

McMullan, M. (2006). Students' perceptions on the use of portfolios in pre-registration nursing education: A questionnaire survey. International Journal of Nursing Studies, 43(3), 333-343.

Mendes, K. D. S., Silveira, R. C. C. P.; Galvão, C. M. (2008). Revisão integrativa: método de pesquisa para a incorporação de evidências na saúde e na enfermagem. Texto Contexto Enfermagem, 17(4), 758-764.

Moher, D. et al. (2009). Preferred reporting items for systematic reviews and meta-analyses: the PRISMA statement. PLOS Medicine, 21(7). https://www.ncbi.nlm.nih.gov/pubmed/19621072.

Parecer $n^{o}$ 3, de 07 de novembro de 2001. (2001). Institui as Diretrizes Curriculares Nacionais do Curso de Graduação em Enfermagem. Brasília, DF: Ministério da Educação e Cultura.

Pascon, D. M., Otrenti, E., \& Mira, V. L. (2018). Percepção e desempenho de graduandos de enfermagem em avaliação de metodologias ativas. Acta Paulista de Enfermagem, 31(1), 61-70.

Pereira, A. S., Shitsuka, D. M., Parreira, F. J., \& Shitsuka, R. (2018). Metodologia da pesquisa científica. https://repositorio.ufsm.br/bitstream/han dle/1/15824/Lic_Computacao_Metodologia-Pesquisa-Cientifica.pdf?sequence=1.

Plakht, Y., Shiyovich, A., Nusbaum, L., \& Raizer, H. (2013). The association of positive and negative feedback with clinical performance, self-evaluation and practice contribution of nursing students. Nurse Education Today, 33(10), 1264-1268.

Reis, D. C. D., Matos, S. S. D., \& Prado, M. L. D. (2005). Representações sociais dos discentes de um curso de graduação em enfermagem sobre avaliação no processo ensino-aprendizagem. Revista Mineira de Enfermagem, 9(4), 328-335.

Ryan, M. (2011). Evaluating portfolio use as a tool for assessment and professional development in graduate nursing education. Journal of Professional Nursing, 27(2), 84-91.

Santos, C. M. D. C., Pimenta, C. A. D. M., \& Nobre, M. R. C. (2007). A estratégia PICO para a construção da pergunta de pesquisa e busca de evidências. Revista Latino-Americana de Enfermagem, 15(3), 508-511.

Santos, J. L. G. et al. (2013). Práticas de enfermeiros na gerência do cuidado em enfermagem e saúde: revisão integrativa. Revista Brasileira de Enfermagem, 66(2), 257-263.

Silva, C. M. D. S. L. M. D., \& Tanji, S. (2008). A avaliação: um desafio que permanece para professores e estudantes de enfermagem. Rev. Enferm. UFPE on line, 2(4), 385-391.

Shiu, A. T., Chan, C. W., Lam, P., Lee, J., \& Kwong, A. N. (2012). Baccalaureate nursing students' perceptions of peer assessment of individual contributions to a group project: a case study. Nurse Education Today, 32(3), 214-218.

Smith, M., \& Rogers, J. (2014). Understanding nursing students' perspectives on the grading of group work assessments. Nurse Education in Practice, 14(2), $112-116$.

Spence, W., \& El-Ansari, W. (2004). Portfolio assessment: practice teachers' early experience. Nurse Education Today, 24(5), 388-401. 
Research, Society and Development, v. 10, n. 3, e18610313238, 2021

(CC BY 4.0) | ISSN 2525-3409 | DOI: http://dx.doi.org/10.33448/rsd-v10i3.13238

Tiwari, A., Lam, D., Yuen, K. H., Chan, R., Fung, T., \& Chan, S. (2005). Student learning in clinical nursing education: Perceptions of the relationship between assessment and learning. Nurse Education Today, 25(4), 299-308.

Tiwari, A., \& Tang, C. (2003). From process to outcome: the effect of portfolio assessment on student learning. Nurse Education Today, 23(4), $269-277$.

Vae, K. J. U., Engström, M., Mårtensson, G., \& Löfmark, A. (2018). A experiência de avaliação de alunos e preceptores de enfermagem durante a prática clínica: um estudo multinível de entrevistas repetidas de díades aluno-preceptor. Formação do Enfermeiro na Prática, 30, 13-19.

Vaismoradi, M., \& Parsa-Yekta, Z. (2011). Iranian nursing students' comprehension and experiences regarding evaluation process: a thematic analysis study. Scandinavian Journal of Caring Sciences, 25(1), 151-159.

Wiebusch, A., \& Lima, V. M. R., (2018). Inovação nas práticas pedagógicas no Ensino Superior: possibilidades para promover o engajamento acadêmico. Educação por escrito, 9(2), 154-169. http://dx..org/10.15448/2179-8435.2018.2.31607. 